\title{
Overcoming clinical inertia in the management of hypertension
}

\author{
Chris Salisbury, Tom Fahey
}

$\infty$

See related article page 1267

$\mathrm{T}$ he term "clinical inertia" is used to summarize 3 related problems associated with inadequate management of chronic diseases: overestimation of care provided; use of "soft" reasons to avoid intensification of therapy; and lack of education, training and practice organization aimed at achieving treatment goals. ${ }^{1}$ Combatting clinical inertia with the aid of health professionals such as nurses and pharmacists following defined treatment algorithms has been seen as a potentially cost-effective way to improve clinical care for patients with chronic conditions such as hypertension and diabetes, particularly in community settings. The randomized controlled trial (RCT) reported by Tobe and colleagues in this issue (page 1267 ) is an example of such a strategy. ${ }^{2}$

In this study, First Nations people with hypertension and type 2 diabetes received intensive and systematic nonpharmacologic management. Those randomly assigned to the intervention group received medication in line with a stepped care protocol administered by a nurse. Those assigned to the control group had follow-up arranged with their primary care physician, who received a summary of clinical practice guidelines that advocated a similar drug treatment regimen.

There was a reduction in mean systolic blood pressure in both groups, with possibly a greater reduction in the intervention group. However, the small size of the study (involving fewer than roo patients) means that the observed difference between the groups may have been due to chance. If there were indeed further benefits for patients in the intervention arm of this study, we cannot tell whether they were due to the provision of care by a nurse rather than by a doctor, or to the rigorous application of a drug treatment algorithm. Although the physicians of patients in the control arm received written guidance about drug treatment, we do not know if they followed it.

A systematic review of studies involving the substitution of nurses for doctors in primary care identified 4 RCTs in which nurses provided routine management for patients with chronic conditions (not specifically hypertension) and in \% which patient outcomes were assessed. ${ }^{3}$ Eight health status outcomes were measured in these studies, of which 1 was significantly better with nurse-led care and 7 showed no significant difference.

The findings of Tobe and colleagues should be viewed in the context of previous RCTs of nurse-led care for patients with hypertension alone ${ }^{4}$ and more recent RCTs of nurse- led care for patients with both diabetes and hypertension..$^{5-7}$ A Cochrane review showed that 7 RCTs of hypertension care led by a health professional other than a doctor (either a pharmacist or nurse) favoured health professional-led care, with improvements in mean systolic and diastolic blood pressure ranging from $\mathrm{I} 3$ to o $\mathrm{mm} \mathrm{Hg}$ systolic and 8 to o $\mathrm{mm}$ $\mathrm{Hg}$ diastolic pressure. ${ }^{4}$ Results from recent RCTs of nurseled care given to patients with both diabetes and hypertension are less convincing, showing reductions in blood pressure in 1 trial, ${ }^{7}$ but no impact on control of blood pressure in 2 other trials, 1 based in hospital clinics and the other in primary care practices. 5,6

\section{New strategies and part- nerships are needed to achieve blood pressure control in rural settings.}

The nature of the interventions in these studies was heterogeneous, so it is not surprising that inconsistent effects were seen. Recommendations differed about the target blood pressure level, which drugs should be used and how often a patient should be reviewed. These conflicting results from RCTs highlight the difficulty in developing and evaluating what is a complex intervention. ${ }^{8}$ Complex interventions provide challenges to researchers when defining, developing, documenting and reproducing the intervention. ${ }^{8}$ In this instance, the nurse-led care developed and evaluated by Tobe and colleagues differed from that in other RCTs in terms of the setting (primary care v. outpatient clinics), the system of health care, the frequency of clinical review, the target blood pressure, and the choice and intensification of antihypertensive drugs used. ${ }^{4-7}$

Considering the findings of Tobe and colleagues along with those from the 2 systematic reviews, it seems that the benefits observed in this new study arise mainly from the application of organized care and a rigorous drug treatment algorithm rather than from the professional background of the 
person providing the care. There is already good evidence that combatting "clinical inertia" by using a vigorous stepped-care approach to hypertension management is likely to have positive results. The landmark Hypertension Detection and Follow Up (HDFP) trial was based on a program of stepped-care drug treatment allied to an emphasis on low cost, convenience and high adherence to therapy. This program led to substantial reductions in blood pressure and lower all-cause mortality after 5 years of follow-up. ${ }^{4}$

The challenge for policy is to ensure that stepped-care programs can be implemented by all health care professionals, including nurses, in a wide range of community settings. As Tobe and colleagues point out, new strategies and partnerships are needed if this is to be achieved in rural settings distant from medical facilities. The challenge for researchers is to disentangle the "active ingredients" of these programs, such as the appropriate training for the care provider and the best treatment regimen. In addition, future studies comparing doctor- and nurse-led care should include an economic evaluation, since ensuring the best use of resources is essential in planning the future organization of services for patients with chronic disease.

Chris Salisbury is Professor of Primary Health Care in the Department of Community Based Medicine, University of Bristol,Cotham House, Cotham Hill, Bristol, UK. Tom Fahey is Professor of Primary Care Medicine in the Division of Community Health Sciences, University of Dundee, Tayside Centre for General Practice, Dundee, UK.
Competing interests: None declared.

Contributors: Both authors contributed to drafting, revising and approving the manuscript.

\section{REFERENCES}

I. Phillips LS, Branch WT, Cook CB, et al. Clinical inertia. Ann Intern Med 200I; I35: 825-34

2. Tobe SW, Pylypchuk G, Wentworth J, et al. Effect of nurse-directed hypertension treatment among First Nations people with existing hypertension and diabetes mellitus: the Diabetes Risk Evaluation and Microalbuminuria (DREAM 3) randomized controlled trial. CMAJ 2006;174(9):1267-7I.

3. Laurant M, Reeves D, Hermens R. Substitution of doctors by nurses in primary care [review]. Cochrane Database Syst Rev 2005;(2):CDooI27I.

4. Fahey T, Schroeder K, Ebrahim S. Interventions used to improve control of blood pressure in patients with hypertension [review]. Cochrane Database Syst Rev 2005; (I):CDoo5182.

5. New JP, Mason JM, Freemantle N, et al. Educational outreach in diabetes to encourage practice nurses to use primary care hypertension and hyperlipidaemia guidelines (EDEN): a randomized controlled trial. Diabet Med 2004; 21:599-603.

6. New JP, Mason JM, Freemantle N, et al. Specialist nurse-led intervention to treat and control hypertension and hyperlipidemia in Diabetes (SPLINT). Diabetes Care 2003;26:2250-4.

7. Denver EA, Barnard M, Woolfson RG, et al. Management of uncontrolled hypertension in a nurse-led clinic compared with conventional care for patients with type 2 diabetes. Diabetes Care 2003; 26:2256-6o.

8. Campbell M, Fitzpatrick R, Haines A, et al. Framework for design and evaluation of complex interventions to improve health. BMJ 2000; 321:694-6.

Correspondence to: Prof. Chris Salisbury, Professor of Primary Health Care, Department of Community Based Medicine, University of Bristol, Cotham House, Cotham Hill, Bristol UK BS66JL; c.salisbury@bristol.ac.uk

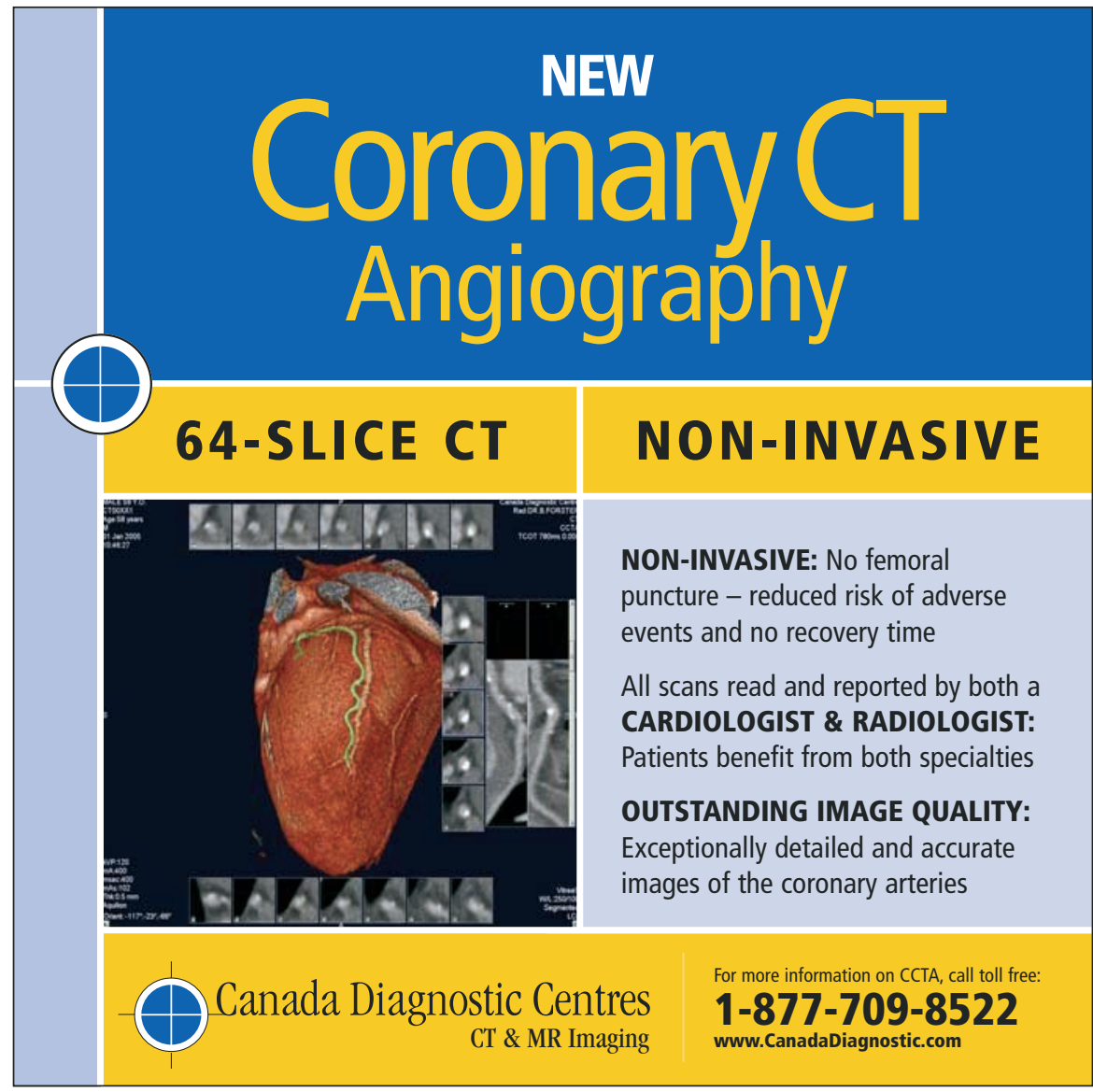

